\title{
A New Approach to Hausdorff Space Theory via the Soft Sets
}

\author{
Güzide Şenel \\ Department of Mathematics, Faculty of Arts and Science, Amasya University, 05100 Amasya, Turkey \\ Correspondence should be addressed to Güzide Şenel; g.senel@amasya.edu.tr \\ Received 1 April 2016; Accepted 8 August 2016 \\ Academic Editor: Anna M. Gil-Lafuente \\ Copyright (C) 2016 Güzide Şenel. This is an open access article distributed under the Creative Commons Attribution License, which \\ permits unrestricted use, distribution, and reproduction in any medium, provided the original work is properly cited. \\ The aim of this paper is to present the concept of soft bitopological Hausdorff space (SBT Hausdorff space) as an original \\ study. Firstly, I introduce some new concepts in soft bitopological space such as SBT point, SBT continuous function, and \\ SBT homeomorphism. Secondly, I define SBT Hausdorff space. I analyse whether a SBT space is Hausdorff or not by SBT \\ homeomorphism defined from a SBT Hausdorff space to researched SBT space. I end my study by defining SBT property and \\ hereditary SBT by SBT homeomorphism and investigate the relations between SBT space and SBT subspace.
}

\section{Introduction}

In applied and theoretical areas of mathematics, we often deal with sets evolved with various structures. However, it may happen that the consideration of a set with classical mathematical approaches is not useful to characterize uncertainty. To overcome these difficulties, Molodtsov [1] introduced the concept of soft set as a new mathematical tool. Later, he developed and applied this theory to several directions [2-4]. New soft set definitions are made, and new classes of soft sets and mappings between different classes of soft sets are studied by many researchers [5-16]. Topology depends strongly on the ideas of set theory. The theory of soft topological spaces is investigated by defining a new soft set theory which can lead to the development of new mathematical models. The topological structure of soft sets also was studied by many authors [7, 11, 17-23] which are defined over an initial universe with a fixed set of parameters.

In 1963, Kelly [24] defined the bitopological space as an original and fundamental work by using two different topologies. It is an extension of general topology. Before Kelly, bitopological space appeared in a narrow sense in [25] as a supplementary work to characterize Baire spaces. In 1990, Ivanov [26] presented a new viewpoint for the theory of bitopological spaces by using a topologic structure on the cartesian product of two sets. There are several works on theory (e.g., [26-31]) and application (e.g., [32-36]) of bitopological spaces.

A soft set with one specific topological structure is not sufficient to develop the theory. In that case, it becomes necessary to introduce an additional structure on the soft set. To confirm this idea, soft bitopological space (SBT) by soft bitopological theory was introduced. In this theory, a soft set was equipped with arbitrary soft topologies.

In this paper, I present the definition of soft bitopological Hausdorff space and construct some basic properties. I introduce the notions of SBT point, SBT continuous function, and SBT homeomorphism. Moreover, I define SBT property and hereditary SBT by SBT homeomorphism and investigate the relations between these concepts.

\section{Preliminaries}

In this section, I will recall the notions of soft sets [1], soft point [23], soft function [37], soft topology [38], bitopological space [24], and soft bitopological space [39]. Then, I will give some properties of these notions.

Throughout this work, $U$ refers to an initial universe, $E$ is a set of parameters, and $P(U)$ is the power set of $U$.

Definition 1 (see [1]). A pair $(f, E)$ is called a soft set (over $U$ ) if and only if $f$ is a mapping or $E$ is the set of all subsets of the set $U$. 
From now on, I will use definitions and operations of soft sets which are more suitable for pure mathematics based on the study of [10].

Definition 2 (see [10]). A soft set $f$ on the universe $U$ is defined by the set of ordered pairs

$$
f=\{(e, f(e)): e \in E\},
$$

where $f: E \rightarrow \mathscr{P}(U)$ such that $f(e)=\emptyset$; if $e \in E \backslash A$, then $f=f_{A}$. $\mathbb{S}$.

Note that the set of all soft sets over $U$ will be denoted by

Definition 3 (see $[10]$ ). Let $f \in \mathbb{S}$. Then,

if $f(e)=\emptyset$ for all $e \in E$, then $f$ is called an empty set, denoted by $\Phi$;

if $f(e)=U$ for all $e \in E$, then $f$ is called universal soft set, denoted by $\widetilde{E}$.

Definition 4 (see $[10])$. Let $f, g \in \mathbb{S}$. Then,

$f$ is a soft subset of $g$, denoted by $f \widetilde{\subseteq} g$, if $f \subseteq g$ for all $e \in E$;

$f$ and $g$ are soft equal, denoted by $f=g$, if and only if $f(e)=g(e)$ for all $e \in E$.

Definition 5 (see [10]). Let $f, g \in \mathbb{S}$. Then, soft union and soft intersection of $f$ and $g$ are defined by the soft sets, respectively:

$$
\begin{aligned}
& f \widetilde{\cup} g=\{f(e) \cup g(e): e \in E\}, \\
& f \widetilde{\cap} g=\{f(e) \cap g(e): e \in E\},
\end{aligned}
$$

and the soft complement of $f$ is defined by

$$
f^{\tilde{c}}=\left\{f(e)^{c}: e \in E\right\}
$$

where $f^{\widetilde{c}}$ is the complement of the set $f(e)$; that is, $f(e)^{c}=$ $U \backslash f_{A}(e)$ for all $e \in E$.

It is easy to see that $\left(f^{\widetilde{c}}\right)^{\widetilde{c}}=f$ and $\Phi^{\widetilde{c}}=\widetilde{E}$.

Proposition 6 (see [10]). Let $f \in \mathbb{S}$. Then,

(i) $f \widetilde{\cup} f=f, f \widetilde{\cap} f=f$;

(ii) $f \widetilde{\cup} \Phi=f, f \widetilde{\cap} \Phi=\Phi$;

(iii) $f \widetilde{\cup} \widetilde{E}=\widetilde{E}, f \widetilde{\cap} \widetilde{E}=f$;

(iv) $f \widetilde{U} f^{\widetilde{c}}=\widetilde{E}, f \widetilde{\cap} f^{\widetilde{c}}=\Phi$.

Proposition 7 (see $[10]$ ). Let $f, g, h \in \mathbb{S}$. Then,

(i) $f \widetilde{\cup} g=g \tilde{U} f, f \widetilde{\cap} g=g \widetilde{\cap} f$;

(ii) $(f \widetilde{\cap} g)^{\tilde{c}}=g^{\widetilde{\mathcal{U}}} \tilde{f^{\tilde{c}}},(f \widetilde{U} g)^{\tilde{c}}=g^{\widetilde{c}} \widetilde{\cap} f^{\widetilde{c}}$; (iii) $(f \widetilde{\cup} g) \widetilde{U} h=f \widetilde{U}(g \widetilde{\cup} h),(f \widetilde{\cap} g) \widetilde{\cap} h=f \widetilde{\cap}(g \widetilde{\cap} h)$;

(iv) $f \widetilde{U}(g \widetilde{\cap} h)=(f \widetilde{U} g) \widetilde{\cap}(f \widetilde{\cup} h)$;

$$
f \widetilde{\cap}(g \widetilde{\cup} h)=(f \widetilde{\cap} g) \widetilde{\cup}(f \widetilde{\cap} h) .
$$

Definition 8 (see [8]). Let $f \in \mathbb{S}$. The power soft set of $f$ is defined by

$$
\mathscr{P}(f)=\left\{f_{i} \widetilde{\subseteq} f: i \in I\right\}
$$

and its cardinality is defined by

$$
|\mathscr{P}(f)|=2^{\sum_{e \in E}|f(e)|},
$$

where $|f(e)|$ is the cardinality of $f(e)$.

Example 9. Let $U=\left\{u_{1}, u_{2}, u_{3}\right\}$ and $E=\left\{e_{1}, e_{2}\right\} . f \in \mathbb{S}$ and

$$
f=\left\{\left(e_{1},\left\{u_{1}, u_{2}\right\}\right),\left(e_{2},\left\{u_{2}, u_{3}\right\}\right)\right\} .
$$

Then,

$$
\begin{aligned}
f_{1} & =\left\{\left(e_{1},\left\{u_{1}\right\}\right)\right\}, \\
f_{2} & =\left\{\left(e_{1},\left\{u_{2}\right\}\right)\right\}, \\
f_{3} & =\left\{\left(e_{1},\left\{u_{1}, u_{2}\right\}\right)\right\}, \\
f_{4} & =\left\{\left(e_{2},\left\{u_{2}\right\}\right)\right\}, \\
f_{5} & =\left\{\left(e_{2},\left\{u_{3}\right\}\right)\right\}, \\
f_{6} & =\left\{\left(e_{2},\left\{u_{2}, u_{3}\right\}\right)\right\}, \\
f_{7} & =\left\{\left(e_{1},\left\{u_{1}\right\}\right),\left(e_{2},\left\{u_{2}\right\}\right)\right\}, \\
f_{8} & =\left\{\left(e_{1},\left\{u_{1}\right\}\right),\left(e_{2},\left\{u_{3}\right\}\right)\right\}, \\
f_{9} & =\left\{\left(e_{1},\left\{u_{1}\right\}\right),\left(e_{2},\left\{u_{2}, u_{3}\right\}\right)\right\}, \\
f_{10} & =\left\{\left(e_{1},\left\{u_{2}\right\}\right),\left(e_{2},\left\{u_{2}\right\}\right)\right\}, \\
f_{11} & =\left\{\left(e_{1},\left\{u_{2}\right\}\right),\left(e_{2},\left\{u_{3}\right\}\right)\right\}, \\
f_{12} & =\left\{\left(e_{1},\left\{u_{2}\right\}\right),\left(e_{2},\left\{u_{2}, u_{3}\right\}\right)\right\}, \\
f_{13} & =\left\{\left(e_{1},\left\{u_{1}, u_{2}\right\}\right),\left(e_{2},\left\{u_{2}\right\}\right)\right\}, \\
f_{14} & =\left\{\left(e_{1},\left\{u_{1}, u_{2}\right\}\right),\left(e_{2},\left\{u_{3}\right\}\right)\right\}, \\
f_{15} & =f, \\
f_{16} & =\Phi
\end{aligned}
$$

are all soft subsets of $f$. So $|\widetilde{P}(f)|=2^{4}=16$.

Definition 10 (see [23]). The soft set $f \in \mathbb{S}$ is called a soft point in $\widetilde{E}$, denoted by $e_{f}$, if there exists an element $e \in E$ such that $f(e) \neq \emptyset$ and $f\left(e^{\prime}\right)=\emptyset$, for all $e^{\prime} \in E \backslash\{e\}$.

Definition 11 (see [23]). The soft point $e_{f}$ is said to belong to a soft set $g \in \mathbb{S}$, denoted by $e_{f} \tilde{\epsilon} g$, if $e \in E$ and $f(e) \subseteq g(e)$. 
Theorem 12 (see [23]). Let $E$ and $U$ be finite sets. The number of all soft points in $f \in \mathbb{S}$ is equal to

$$
\sum_{e \in E}\left(2^{|f(e)|}-1\right)
$$

Theorem 13 (see [23]). A soft set can be written as the soft union of all its soft points.

Theorem 14 (see [23]). Let $f, g \in \mathbb{S}$. Then,

$$
e_{i_{f}} \tilde{\epsilon} g \Longrightarrow f \widetilde{\subseteq} g
$$

for all $e_{i_{f}} \tilde{\epsilon} f$.

Definition 15 (see [37]). (i) Let $X \subseteq E$ and $f \in S_{X}(U)$ be a soft set in $\mathbb{S}$. The image of $f$ under $\varphi_{\psi}$ is a soft set in $S_{K}(V)$ such that

$$
\begin{aligned}
\varphi_{\psi}(f)\left(k_{j}\right) \\
= \begin{cases}\bigcup_{e_{i} \in \psi^{-1}\left(k_{j}\right) \cap X} \varphi\left(f\left(e_{i}\right)\right), & \psi^{-1}\left(k_{j}\right) \cap X \neq \emptyset \\
\emptyset, & \psi^{-1}\left(k_{j}\right) \cap X=\emptyset\end{cases}
\end{aligned}
$$

for all $k_{j} \in K$.

(ii) Let $Y \subseteq K$ and $g \in S_{Y}(V)$. Then, the inverse image of $g$ under $\varphi_{\psi}$ is a soft set in $S_{E}(U)$ such that

$$
\varphi_{\psi}^{-1}(g)\left(e_{i}\right)= \begin{cases}\varphi^{-1}\left(g\left(\psi\left(e_{i}\right)\right)\right), & \psi\left(e_{i}\right) \in Y \\ \emptyset, & \psi\left(e_{i}\right) \notin Y\end{cases}
$$

for all $e_{i} \in E$.

Definition 16 (see [38]). Let $\Phi \neq X \subseteq E$ and $f \in \mathbb{S}$. Let $\tilde{\tau}=\left\{g_{i}\right\}_{i \in I}$ be the collection of soft sets over $f$. Then, $\widetilde{\tau}$ is called a soft topology on $f$ if $\tilde{\tau}$ satisfies the following axioms:

(i) $\Phi, f \in \tilde{\tau}$,

(ii) $\left\{g_{i}\right\}_{i \in I} \subseteq \widetilde{\tau} \Rightarrow \widetilde{\bigcup}_{i \in I} g_{i} \in \widetilde{\tau}$,

(iii) $\left\{g_{i}\right\}_{i=1}^{n} \subseteq \tilde{\tau} \Rightarrow \widetilde{\bigcap}_{i=1}^{n} g_{i} \in \tilde{\tau}$.

The pair $(f, \tilde{\tau})$ is called a soft topological space over $f$ and the members of $\widetilde{\tau}$ are said to be soft open in $f$.

Example 17. Let us consider the soft subsets of $f$ that are given in Example 9. Then, $\tilde{\tau}^{1}=\widetilde{\mathscr{P}}(f), \tilde{\tau}^{0}=\{\Phi, f\}$, and $\widetilde{\tau}=\left\{\Phi, f, f_{2}, f_{11}, f_{13}\right\}$ are some soft topologies on $f$.

Definition 18 (see [38]). Let $(f, \widetilde{\tau})$ and $g \in \mathbb{S}$. Then, $g$ is soft closed in $\tilde{\tau}$ if $g^{\tilde{c}} \in \tilde{\tau}$.

Definition 19 (see [24]). Let $X \neq \emptyset$, and let $\tau_{1}$ and $\tau_{2}$ be two different topologies on $X$. Then, $\left(X, \tau_{1}, \tau_{2}\right)$ is called a bitopological space. Throughout this paper, $\left(X, \tau_{1}, \tau_{2}\right)$ [or simply $X$ ] denote bitopological space on which no seperation axioms are assumed unless explicitly stated.
Definition 20 (see [24]). A subset $S$ of $X$ is called $\tau_{1} \tau_{2}$-open if $S \in \tau_{1} \cup \tau_{2}$ and the complement of $\tau_{1} \tau_{2}$-open is $\tau_{1} \tau_{2}$-closed.

Example 21. Let $X=\{a, b, c\}, \tau_{1}=\{\emptyset, X,\{a\}\}$, and $\tau_{2}=$ $\{\emptyset, X,\{b\}\}$. The sets in $\{\emptyset, X,\{a\},\{b\},\{a, b\}\}$ are called $\tau_{1} \tau_{2}$ open and the sets in $\{\emptyset, X,\{b, c\},\{a, c\},\{c\}\}$ are called $\tau_{1} \tau_{2}$ closed.

Definition 22 (see [24]). Let $S$ be a subset of $X$. Then,

(i) the $\tau_{1} \tau_{2}$-interior of $S$, denoted by $\tau_{1} \tau_{2} \operatorname{int}(S)$, is defined by

$$
\bigcup\left\{F: S \subset F, F \text { is a } \tau_{1} \tau_{2} \text {-open }\right\}
$$

(ii) the $\tau_{1} \tau_{2}$-closure of $S$, denoted by $\tau_{1} \tau_{2} \operatorname{cl}(S)$, is defined by

$$
\bigcap\left\{F: S \subset F, F \text { is a } \tau_{1} \tau_{2} \text {-closed }\right\}
$$

Definition 23 (see [39]). Let $f$ be a nonempty soft set on the universe $U$, and let $\widetilde{\tau}_{1}$ and $\widetilde{\tau}_{2}$ be two different soft topologies on $f$. Then, $\left(f, \tilde{\tau}_{1}, \tilde{\tau}_{2}\right)$ is called a soft bitopological space which is abbreviated as SBT space.

Definition 24 (see [39]). Let $\left(f, \widetilde{\tau}_{1}, \widetilde{\tau}_{2}\right)$ be a SBT space and $g \tilde{\subset} f$. Then, $g$ is called $\tilde{\tau}_{1} \tilde{\tau}_{2}$-soft open if $g=h \widetilde{\cup} k$, where $h \in \widetilde{\tau}_{1}$ and $k \in \widetilde{\tau}_{2}$.

The soft complement of $\widetilde{\tau}_{1} \widetilde{\tau}_{2}$-soft open set is called $\tilde{\tau}_{1} \widetilde{\tau}_{2}$ soft closed.

Definition 25 (see [39]). Let $g$ be a soft subset $f$. Then, $\widetilde{\tau}_{1} \widetilde{\tau}_{2}$ interior of $g$, denoted by $(g)_{\tilde{\tau}_{1} \tau_{2}}^{\circ}$, is defined by the following:

$$
(g)_{\widetilde{\tau}_{1} \widetilde{\tau}_{2}}^{\circ}=\widetilde{\bigcup}\left\{g: h \widetilde{\subset} g, h \text { is } \tilde{\tau}_{1} \tilde{\tau}_{2} \text {-soft open }\right\} .
$$

The $\widetilde{\tau}_{1} \widetilde{\tau}_{2}$-closure of $g$, denoted by $(\bar{g})_{\widetilde{\tau}_{1}} \widetilde{\tau}_{2}$, is defined by the following:

$$
(\bar{g})_{\widetilde{\tau}_{1} \widetilde{\tau}_{2}}=\widetilde{\bigcap}\left\{h: g \widetilde{c} h, h \text { is } \widetilde{\tau}_{1} \widetilde{\tau}_{2} \text {-soft closed }\right\} .
$$

Note that $(g)_{\tilde{\tau}_{1}}^{\circ} \tau_{2}$ is the biggest $\tilde{\tau}_{1} \widetilde{\tau}_{2}$-soft open set contained in $g$ and $(\bar{g})_{\tau_{1}} \tilde{\tau}_{2}$ is the smallest $\tilde{\tau}_{1} \tilde{\tau}_{2}$-soft closed set contained in $g$.

Example 26 (see [39]). Considering Example 9, $\tilde{\tau}_{1}=$ $\left\{\Phi, f, f_{2}\right\}$ and $\tilde{\tau}_{2}=\left\{\Phi, f, f_{1}, f_{4}\right\}$. Then, $\left\{\Phi, f, f_{1}, f_{2}, f_{3}, f_{4}\right\}$ are $\widetilde{\tau}_{1} \widetilde{\tau}_{2}$-soft open sets and $\left\{\Phi, f, f_{1}, f_{2}, f_{5}\right\}$ are $\widetilde{\tau}_{1} \tilde{\tau}_{2}$-soft closed sets.

\section{SBT Hausdorff Space}

In this section, I present the definition of soft bitopological Hausdorff space and construct some basic properties. I introduce the notions of SBT point, SBT continuous function, and SBT homeomorphism. I analyse whether a SBT space is Hausdorff or not by SBT homeomorphism defined from a SBT Hausdorff space to researched SBT space. Moreover, I define SBT property and hereditary SBT by SBT homeomorphism and investigate the relations between these concepts. 
Definition 27. Let $\left(f, \tilde{\tau}_{1}, \tilde{\tau}_{2}\right)$ be a SBT space and $g \tilde{\subset} f$. Then, $g$ is called $\widetilde{\tau}_{1} \widetilde{\tau}_{2}$-soft point if $g$ is a soft point in $\mathbb{S}$ and is denoted by $e_{g} \tilde{\epsilon} f$.

Definition 28. Let $\left(f, \widetilde{\tau}_{1}, \widetilde{\tau}_{2}\right)$ be a SBT space and let $g$ be a soft set over $U$. The soft point $e_{f} \tilde{\in} \mathbb{S}$ is called a $\tilde{\tau}_{1} \widetilde{\tau}_{2}$-interior point of a soft set $g$ if there exists a soft open set $h$ such that $e_{f} \tilde{\epsilon} h \tilde{\epsilon} g$.

Definition 29. Let $\left(f, \widetilde{\tau}_{1}, \widetilde{\tau}_{2}\right)$ and $\left(g, \tilde{\tau}_{1}^{*}, \tilde{\tau}_{2}^{*}\right)$ be two SBT spaces and $\varphi_{\psi}: S_{E}(U) \rightarrow S_{K}(V)$ be a soft function. If $\varphi_{\psi}^{-1}(h) \tilde{\epsilon} \tilde{\tau}_{1}$ for all $h \tilde{\epsilon} \widetilde{\tau}_{1}^{*}$ and $\varphi_{\psi}^{-1}(k) \tilde{\epsilon} \widetilde{\tau}_{2}$ for all $k \tilde{\epsilon} \widetilde{\tau}_{2}^{*}$, then $\varphi_{\psi}$ soft function is called $\widetilde{\tau}_{1} \widetilde{\tau}_{2}$ continuous function.

Definition 30. Let $\left(f, \tilde{\tau}_{1}, \widetilde{\tau}_{2}\right)$ and $\left(g, \tilde{\tau}_{1}^{*}, \tilde{\tau}_{2}^{*}\right)$ be two SBT spaces and $\varphi_{\psi}: S_{E}(U) \rightarrow S_{K}(V)$ be a soft function and $e_{f} \tilde{\epsilon} f$.

(i) $\varphi_{\psi}$ soft function is $\tilde{\tau}_{1} \tilde{\tau}_{2}$ continuous function at $e_{f} \tilde{\epsilon} f$ if, for each $g \tilde{\epsilon} k, \varphi_{\psi}\left(e_{f}\right) \tilde{\epsilon} k \tilde{\epsilon} \widetilde{\tau}_{1}^{*} \widetilde{\cup} \widetilde{\tau}_{2}^{*}$, there exists $h \tilde{\epsilon} t$, $e_{f} \tilde{\epsilon} t \tilde{\in} \widetilde{\tau}_{1} \tilde{\cup} \widetilde{\tau}_{2}$, such that $\varphi_{\psi}\left(e_{f}\right) \widetilde{\subseteq} g$.

(ii) $\varphi_{\psi}$ is $\widetilde{\tau}_{1} \widetilde{\tau}_{2}$ continuous on $f$ if $\varphi_{\psi}$ is soft continuous at each soft point in $f$.

Definition 31. A soft function $\varphi_{\psi}: S_{E}(U) \rightarrow S_{K}(V)$ between two SBT spaces $\left(f, \tilde{\tau}_{1}, \tilde{\tau}_{2}\right)$ and $\left(g, \tilde{\tau}_{1}^{*}, \tilde{\tau}_{2}^{*}\right)$ is called a SBT homeomorphism if it has the following properties:

(i) $\varphi_{\psi}$ is a soft bijection (soft surjective and soft injective).

(ii) $\varphi_{\psi}$ is $\widetilde{\tau}_{1} \widetilde{\tau}_{2}$ continuous.

(iii) $\varphi_{\psi}^{-1}$ is $\widetilde{\tau}_{1} \tilde{\tau}_{2}$ continuous.

A soft function with these three properties is called $\widetilde{\tau}_{1} \widetilde{\tau}_{2}$ homeomorphism. If such a soft function exists, we say $\left(f, \widetilde{\tau}_{1}, \widetilde{\tau}_{2}\right)$ and $\left(g, \widetilde{\tau}_{1}^{*}, \widetilde{\tau}_{2}^{*}\right)$ are SBT homeomorphic.

Definition 32. SBT property is a property of a SBT space which is invariant under SBT homeomorphisms.

That is, a property of SBT spaces is a SBT property if whenever a SBT space possesses that property every space SBT homeomorphic to this space possesses that property.

Definition 33. Let $\left(f, \widetilde{\tau}_{1}, \widetilde{\tau}_{2}\right)$ be a SBT space. If for each pair of distinct soft points $e_{i_{f_{i}}}, e_{j_{f_{j}}} \tilde{\epsilon} f$ there exist a $\widetilde{\tau}_{1}$ open set $g$ and $\tilde{\tau}_{2}$ open set $h$ such that $e_{i_{f_{i}}} \tilde{\epsilon} g, e_{j_{f_{j}}} \tilde{\epsilon} h$, and $g \widetilde{\cap} h=\Phi$, then $\left(f, \widetilde{\tau}_{1}, \widetilde{\tau}_{2}\right)$ is called a SBT Hausdorff space.

Example 34. Let $f=\left\{\left(e_{1},\left\{u_{1}, u_{2}\right\}\right),\left(e_{2},\left\{u_{2}, u_{3}\right\}\right)\right\}$, $\tilde{\tau}_{1}=$ $\left\{\Phi, f,\left(e_{1},\left\{u_{1}\right\}\right),\left(e_{1},\left\{u_{2}\right\}\right)\right\}$, and $\widetilde{\tau}_{2}=\left\{\Phi, f,\left(e_{2},\left\{u_{2}\right\}\right)\right\}$.

Then, $\widetilde{\tau}_{1} \widetilde{\tau}_{2}$-soft open sets are

$$
\left\{\Phi, f,\left(e_{1},\left\{u_{1}\right\}\right),\left(e_{1},\left\{u_{2}\right\}\right),\left(e_{2},\left\{u_{2}\right\}\right),\left(e_{1},\left\{u_{1}, u_{2}\right\}\right)\right\}
$$

Let $e_{1_{f_{1}}}=\left\{\left(e_{1},\left\{u_{1}\right\}\right)\right\}, e_{1_{f_{2}}}=\left\{\left(e_{1},\left\{u_{2}\right\}\right)\right\}$, and $e_{1_{f_{1}}} \neq e_{1_{f_{2}}}$.

$g_{1}=\left\{\left(e_{1},\left\{u_{1}\right\}\right)\right\}, g_{2}=\left\{\left(e_{1},\left\{u_{2}\right\}\right)\right\}, e_{1_{f_{1}}} \tilde{\epsilon} g_{1}, e_{1_{f_{2}}} \tilde{\epsilon} g_{2}$, and $g_{1} \widetilde{\cap} g_{2}=\Phi$.

Hence, $\left(f, \widetilde{\tau}_{1}, \widetilde{\tau}_{2}\right)$ is a SBT Hausdorff space.

\section{More on SBT Hausdorff Space}

We continue the study of the theory of SBT Hausdorff spaces. In order to investigate all the soft bitopological modifications of SBT Hausdorff spaces, I present new definitions of $\widetilde{\tau}_{1} \widetilde{\tau}_{2}$ soft closure, SBT homeomorphism, SBT property, and hereditary SBT. I have explored relations between SBT space and SBT subspace by hereditary SBT.

Definition 35. Let $\left(f, \widetilde{\tau}_{1}, \widetilde{\tau}_{2}\right)$ be a SBT space and $\widetilde{\mathscr{B}}_{\widetilde{\tau}_{1} \tilde{\tau}_{2}} \widetilde{\subseteq} \widetilde{\tau}_{1} \widetilde{\cup} \widetilde{\tau}_{2}$. If every element of $\widetilde{\tau}_{1} \widetilde{\cup} \widetilde{\tau}_{2}$ can be written as the union of elements of $\widetilde{\mathscr{B}}_{\widetilde{\tau}_{1} \tau_{2}}$, then $\widetilde{\mathscr{B}}_{\tau_{1} \tau_{2}}$ is called $\widetilde{\tau}_{1} \widetilde{\tau}_{2}$-soft basis for $\left(f, \widetilde{\tau}_{1}, \widetilde{\tau}_{2}\right)$.

Each element of $\widetilde{\mathscr{B}}_{\widetilde{\tau}_{1} \widetilde{\tau}_{2}}$ is called soft bitopological basis element.

Theorem 36. Let $\left(f, \widetilde{\tau}_{1}, \widetilde{\tau}_{2}\right)$ be a SBT space and $\widetilde{\mathscr{B}}_{\widetilde{\tau}_{1} \widetilde{\tau}_{2}}$ be a soft basis for $\left(f, \widetilde{\tau}_{1}, \widetilde{\tau}_{2}\right)$. Then, $\widetilde{\tau}_{1} \cup \widetilde{\tau}_{2}$ equals the collections of all soft unions of elements $\widetilde{\mathscr{B}}_{\widetilde{\tau}_{1} \widetilde{\tau}_{2}}$.

Proof. It is clearly seen from Definition 35.

Theorem 37. Every finite point $\widetilde{\tau}_{1} \widetilde{\tau}_{2}$-soft set in a SBT Hausdorff space is $\widetilde{\tau}_{1} \tilde{\tau}_{2}$-soft closed set.

Proof. Let $\left(f, \widetilde{\tau}_{1}, \widetilde{\tau}_{2}\right)$ be a SBT Hausdorff space. It suffices to show that every soft point $\left\{e_{f}\right\}$ is $\widetilde{\tau}_{1} \widetilde{\tau}_{2}$-soft closed. If $e_{g}$ is a soft point of $f$ different from $e_{f}$, then $e_{f}$ and $e_{g}$ have disjoint $\tilde{\tau}_{1} \tilde{\tau}_{2}-$ soft neighborhoods $g_{1}$ and $g_{2}$, respectively. Since $g_{1}$ does not soft-intersect $\left\{e_{g}\right\}$, the soft point $e_{f}$ cannot belong to the $\widetilde{\tau}_{1} \widetilde{\tau}_{2}$ soft closure of the set $\left\{e_{g}\right\}$. As a result, the $\widetilde{\tau}_{1} \widetilde{\tau}_{2}$-soft closure of the set $\left\{e_{f}\right\}$ is $\left\{e_{f}\right\}$ itself, so that it is $\tilde{\tau}_{1} \tilde{\tau}_{2}$-soft closed.

In order to show Theorem 37, we have the following example.

Example 38. Consider the SBT Hausdorff space in Example 34. Define finite soft point $\widetilde{\tau}_{1} \widetilde{\tau}_{2}$-soft sets $f_{1}=\left\{\left(e_{1},\left\{u_{1}\right\}\right)\right\}$ and $f_{2}=\left\{\left(e_{1},\left\{u_{2}\right\}\right)\right\}$ such that soft points are $e_{1_{f_{1}}}=$ $\left\{\left(e_{1},\left\{u_{1}\right\}\right)\right\}$ and $e_{1_{f_{2}}}=\left\{\left(e_{1},\left\{u_{2}\right\}\right)\right\}$. By taking account of the notion that $e_{1_{f_{2}}}$ is a soft point of $f$ different from $e_{1_{f_{1}}}$, then $e_{1_{f_{1}}}$ and $e_{1_{f_{2}}}$ have disjoint $\widetilde{\tau}_{1} \widetilde{\tau}_{2}$-soft neighborhoods $g_{1}$ and $g_{2}$ such that

$$
\begin{aligned}
& g_{1}=\left\{\left(e_{1},\left\{u_{1}\right\}\right)\right\}, \\
& f_{2}=\left\{\left(e_{1},\left\{u_{2}\right\}\right)\right\} .
\end{aligned}
$$

Since $\left\{\left(e_{1},\left\{u_{1}\right\}\right)\right\} \widetilde{\cap}\left\{\left(e_{1},\left\{u_{2}\right\}\right)\right\}=\Phi, \widetilde{\tau}_{1} \widetilde{\tau}_{2}$-soft closure of the set $e_{1_{1}}$ is itself, so that it is $\widetilde{\tau}_{1} \widetilde{\tau}_{2}$-soft closed.

Theorem 39. If $\left(f, \widetilde{\tau}_{1}, \widetilde{\tau}_{2}\right)$ is a SBT Hausdorff space and $\varphi_{\psi}$ : $S_{E}(U) \rightarrow S_{K}(V)$ between two SBT spaces $\left(f, \tilde{\tau}_{1}, \tilde{\tau}_{2}\right)$ and $\left(g, \widetilde{\tau}_{1}^{*}, \widetilde{\tau}_{2}^{*}\right)$ is a SBT homeomorphism, then $\left(g, \widetilde{\tau}_{1}^{*}, \widetilde{\tau}_{2}^{*}\right)$ is a SBT Hausdorff space.

Proof. Let $e_{1_{g_{1}}}, e_{2_{g_{2}}} \tilde{\epsilon} g$ such that $e_{1_{g_{1}}} \neq e_{2_{g_{2}}}$. Since $\varphi_{\psi}$ is soft surjective, there exist $e_{1_{f_{1}}}, e_{2_{f_{2}}} \tilde{\epsilon} f$ such that $\varphi_{\psi}\left(e_{1_{f_{1}}}\right)=$ 
$e_{g_{g_{1}}}, \varphi_{\psi}\left(e_{2_{f_{2}}}\right)=e_{2_{g_{2}}}$, and $e_{1_{f_{1}}} \neq e_{2_{f_{2}}}$. From the hypothesis, $\left(f, \widetilde{\tau}_{1}, \widetilde{\tau}_{2}\right)$ is a SBT Hausdorff space, so there exist $h, k \tilde{\epsilon} \widetilde{\tau}_{1} \widetilde{\cup} \widetilde{\tau}_{2}$ such that $e_{1_{f_{1}}} \tilde{\epsilon} h, e_{f_{f_{2}}} \tilde{\epsilon} k$, and $h \widetilde{\cap} k=\Phi$. For each $e \tilde{\epsilon} E, e_{1_{f_{1}}} \tilde{\epsilon} h(e), e_{2_{f_{2}}} \tilde{\epsilon} k(e)$, and $h(e) \cap k(e)=\emptyset$. So, $\varphi_{\psi}\left(e_{1_{f_{1}}}\right)=e_{1_{g_{1}}} \tilde{\epsilon} \varphi_{\psi}(h(e))$ and $\varphi_{\psi}\left(e_{f_{f_{2}}}\right)=e_{2_{g_{2}}} \tilde{\epsilon} \varphi_{\psi}(k(e))$. Hence, $e_{1_{g_{1}}} \tilde{\epsilon} \varphi_{\psi}(h), e_{2_{g_{2}}} \tilde{\epsilon} \varphi_{\psi}(k)$. Since $\varphi_{\psi}$ is soft open, then $\varphi_{\psi}(h), \varphi_{\psi}(k) \tilde{\epsilon} \widetilde{\tau}_{1}^{*} \tilde{\mathrm{U}} \widetilde{\tau}_{2}^{*}$ and since $\varphi_{\psi}$ is soft injective, $\varphi_{\psi}(h) \tilde{\cap} \varphi_{\psi}(k)=\varphi_{\psi}(h \widetilde{\cap} k)=\Phi$. Thus, $\left(g, \widetilde{\tau}_{1}^{*}, \tilde{\tau}_{2}^{*}\right)$ is a SBT Hausdorff space.

From Definition 32 and Theorem 39, we have the following.

Remark 40. The property of being SBT Hausdorff space is a SBT property.

Theorem 41. Let $\left(f, \widetilde{\tau}_{1}, \widetilde{\tau}_{2}\right)$ be a SBT space and $g \widetilde{\subseteq} f$. Then, collections

$$
\begin{aligned}
& \tilde{\tau}_{1_{g}}=\left\{g_{i} \tilde{\cap} g: g_{i} \in \tilde{\tau}_{1}, i \in I \widetilde{\subseteq} \mathbb{N}\right\}, \\
& \tilde{\tau}_{2_{g}}=\left\{h_{i} \widetilde{\cap} g: h_{i} \in \widetilde{\tau}_{2}, i \in I \widetilde{\subseteq} \mathbb{N}\right\}
\end{aligned}
$$

are soft bitopologies on $\mathrm{g}$.

Proof. Indeed, the union of the soft topologies contains $\Phi$ and $g$ because $\Phi \widetilde{\cap} g=\Phi$ and $f \widetilde{\cap} g=g$, where $\widetilde{\tau}_{1} \widetilde{\cup} \widetilde{\tau}_{2}=\left\{g_{i} \widetilde{\cup} h_{i}\right.$ : $\left.g_{i} \widetilde{\cup} h_{i} \widetilde{\subseteq} f, i \in I\right\}, \widetilde{\tau}_{1} \widetilde{\cup} \widetilde{\tau}_{2}=\left\{g_{i} \widetilde{\cup} h_{i}: g_{i} \widetilde{\cup} h_{i} \widetilde{\simeq} f, i \in I\right\} ;$ it is closed under finite soft intersections and arbitrary soft unions:

$$
\begin{aligned}
& \bigcap_{i=1}^{n}\left(g_{i} \widetilde{\cap} g\right)=\left(\bigcap_{i=1}^{n} g_{i}\right) \tilde{n} g, \\
& \widetilde{\bigcup}{ }_{i \in I}\left(g_{i} \widetilde{\cap} g\right)=\left(\widetilde{\left.\bigcup_{i \in I} g_{i}\right) \tilde{n} g .}\right.
\end{aligned}
$$

In order to show Theorem 41, we have the following example.

Example 42. Let us consider the soft subsets of $f$ that are given in Example 9. Then, $\widetilde{\tau}^{1}=\widetilde{\mathscr{P}}(f), \widetilde{\tau}^{0}=\{\Phi, f\}$, and $\widetilde{\tau}=\left\{\Phi, f, f_{2}, f_{11}, f_{13}\right\}$ are some soft topologies on $f$.

By taking account of $g=f_{9}$, then $\widetilde{\tau}_{g}=\left\{\Phi, f_{5}, f_{7}, f_{9}\right\}$, and so $\left(g, \widetilde{\tau}_{g}\right)$ is a soft topological subspace of $(f, \widetilde{\tau})$. Hence, we get that $\left(g, \widetilde{\tau}_{1_{g}}, \widetilde{\tau}_{2_{g}}\right)$ is a soft bitopological space on $g$.

Definition 43. Let $\left(f, \widetilde{\tau}_{1}, \widetilde{\tau}_{2}\right)$ be a SBT space and $g \widetilde{c} f$. If collections $\widetilde{\tau}_{1_{g}}=\left\{g_{i} \tilde{\cap} g: g_{i} \in \widetilde{\tau}_{1}, i \in I \widetilde{\subseteq} \mathbb{N}\right\}$ and $\tilde{\tau}_{2_{g}}=$ $\left\{h_{i} \tilde{\cap} g: h_{i} \in \widetilde{\tau}_{2}, i \in I \widetilde{\subseteq} \mathbb{N}\right\}$ are two soft topologies on $g$, then a SBT space $\left(g, \widetilde{\tau}_{1 g}, \widetilde{\tau}_{2_{g}}\right)$ is called a SBT subspace of $\left(f, \widetilde{\tau}_{1}, \widetilde{\tau}_{2}\right)$.

In order to show Definition 43, we have the following example.
Example 44. By taking account of Example 42 and considering that $\left(f, \widetilde{\tau}_{1}, \widetilde{\tau}_{2}\right)$ is a SBT Hausdorff space ordered by inclusion, we have that $\left(g, \widetilde{\tau}_{1_{g}}, \widetilde{\tau}_{2_{g}}\right)$ is called a SBT Hausdorff space of $\left(f, \widetilde{\tau}_{1}, \widetilde{\tau}_{2}\right)$.

Theorem 45. Every SBT open set in $\left(f, \widetilde{\tau}_{1}, \widetilde{\tau}_{2}\right)$ is SBT open in SBT subspace of $\left(f, \widetilde{\tau}_{1}, \widetilde{\tau}_{2}\right)$.

Proof. It is clearly seen from Definition 43.

Theorem 46. Let $\left(f, \widetilde{\tau}_{1}, \widetilde{\tau}_{2}\right)$ be a SBT Hausdorff space and $g \tilde{\subset} f$. Then, $\left(g, \widetilde{\tau}_{1_{g}}, \widetilde{\tau}_{2_{g}}\right)$ is a SBT Hausdorff space.

Proof. Let $e_{1_{g_{1}}}, e_{2_{g_{2}}} \tilde{\epsilon} g$ such that $e_{1_{g_{1}}} \neq e_{2_{g_{2}}}$. From the hypothesis, $g \widetilde{\subset} f$, so $e_{g_{1}}, e_{2_{g_{2}}} \tilde{\epsilon} f$. Since $\left(f, \widetilde{\tau}_{1}, \widetilde{\tau}_{2}\right)$ is a SBT Hausdorff space, there exist $h, k \tilde{\epsilon} \widetilde{\tau}_{1} \widetilde{\cup} \widetilde{\tau}_{2}$ such that $e_{1_{f_{1}}} \tilde{\epsilon} h$, $e_{2_{f_{2}}} \tilde{\epsilon} k$ and $h \tilde{\cap} k=\Phi$. So $e_{1_{g_{1}}} \tilde{\epsilon} h \widetilde{\cap} g$ and $e_{2_{g_{2}}} \tilde{\epsilon} k \tilde{\cap} g$.

$$
(h \tilde{\cap} g) \widetilde{\cap}(k \widetilde{\cap} g)=(h \widetilde{\cap} k) \tilde{\cap} g=\Phi .
$$

Thus, $\left(g, \widetilde{\tau}_{1_{g}}, \widetilde{\tau}_{2_{g}}\right)$ is SBT Hausdorff space.

From Definition 43 and Theorem 46, we have the following.

Remark 47. The property of being a soft SBT Hausdorff space is hereditary.

\section{Conclusion}

A soft set with one specific topological structure is not sufficient to develop the theory. In that case, it becomes necessary to introduce an additional structure on the soft set. To confirm this idea, soft bitopological space (SBT) by soft bitopological theory was introduced. It makes it more flexible to develop the theory of soft topological spaces with its applications. Thus, in this paper, I make a new approach to the SBT space theory.

In the present work, I introduce the concept of soft bitopological Hausdorff space (SBT Hausdorff space) as an original study. Firstly, I introduce some new concepts in soft bitopological space such as SBT point, SBT continuous function, and SBT homeomorphism. Secondly, I define SBT Hausdorff space. I analyse whether a SBT space is Hausdorff or not by SBT homeomorphism defined from a SBT Hausdorff space to researched SBT space. In order to investigate all the soft bitopological modifications of SBT Hausdorff spaces, I present new definitions of $\widetilde{\tau}_{1} \widetilde{\tau}_{2}$-soft closure, SBT homeomorphism, SBT property, and hereditary SBT. I have explored relations between SBT space and SBT subspace by hereditary SBT.

I hope that findings in this paper will be useful to characterize the SBT Hausdorff spaces; some further works can be done on the properties of hereditary SBT and SBT property to carry out a general framework for applications of SBT spaces. 


\section{Competing Interests}

The author declares that there are no competing interests.

\section{References}

[1] D. Molodtsov, "Soft set theory-first results," Computers \& Mathematics with Applications, vol. 37, no. 4-5, pp. 19-31, 1999.

[2] D. A. Molodtsov, "The description of a dependence with the help of soft sets," Journal of Computer and Systems Sciences International, vol. 40, no. 6, pp. 977-984, 2001.

[3] D. Molodtsov, The Theory of Soft Sets, URSS Publishers, Moscow, Russia, 2004 (Russian).

[4] D. A. Molodtsov, V. Y. Leonov, and D. V. Kovkov, "Soft sets technique and its application," Nechetkie Sistemy i Myagkie Vychisleniya, vol. 1, no. 1, pp. 8-39, 2006.

[5] H. Aktaş and N. Çağman, "Soft sets and soft groups," Information Sciences, vol. 177, no. 13, pp. 2726-2735, 2007.

[6] M. I. Ali, F. Feng, X. Liu, W. K. Min, and M. Shabir, "On some new operations in soft set theory," Computers \& Mathematics with Applications, vol. 57, no. 9, pp. 1547-1553, 2009.

[7] A. Aygünoğlu and H. Aygün, "Some notes on soft topological spaces," Neural Computing and Applications, vol. 21, supplement 1, pp. 113-119, 2011.

[8] N. Çağman and S. Enginoğlu, "Soft matrix theory and its decision making," Computers \& Mathematics with Applications, vol. 59, no. 10, pp. 3308-3314, 2010.

[9] N. Çağman and S. Enginoğlu, "Soft set theory and uni-int decision making," European Journal of Operational Research, vol. 207, pp. 848-855, 2010.

[10] N. Çağman, "Contributions to the theory of soft sets," Journal of New Result in Science, vol. 4, pp. 33-41, 2014.

[11] D. N. Georgiou and A. C. Megaritis, "Soft set theory and topology," Applied General Topology, vol. 15, no. 1, pp. 93-109, 2014.

[12] O. Kazanci, S. Yilmaz, and S. Yamak, "Soft sets and soft BCHalgebras," Hacettepe Journal of Mathematics and Statistics, vol. 39, no. 2, pp. 205-217, 2010.

[13] P. K. Maji, R. Biswas, and A. R. Roy, "Soft set theory," Computers \& Mathematics with Applications, vol. 45, no. 4-5, pp. 555-562, 2003.

[14] P. K. Maji, A. R. Roy, and R. Biswas, "An application of soft sets in a decision making problem," Computers \& Mathematics with Applications, vol. 44, no. 8-9, pp. 1077-1083, 2002.

[15] P. K. Maji, R. Biswas, and A. R. Roy, "Fuzzy soft sets," Journal of Fuzzy Mathematics, vol. 9, no. 3, pp. 589-602, 2001.

[16] Y. Zou and Z. Xiao, "Data analysis approaches of soft sets under incomplete information," Knowledge-Based Systems, vol. 21, no. 8, pp. 941-945, 2008.

[17] K. V. Babitha and J. J. Sunil, "Soft set relations and functions," Computers \& Mathematics with Applications, vol. 60, no. 7, pp. 1840-1849, 2010.

[18] E. F. Lashin, A. M. Kozae, A. A. Abo Khadra, and T. Medhat, "Rough set theory for topological spaces," International Journal of Approximate Reasoning, vol. 40, no. 1-2, pp. 35-43, 2005.

[19] W. K. Min, "A note on soft topological spaces," Computers \& Mathematics with Applications, vol. 62, no. 9, pp. 3524-3528, 2011.

[20] E. Peyghan, B. Samadi, and A. Tayebi, "About soft topological spaces," Journal of New Results in Science, vol. 2, pp. 60-75, 2013.
[21] G. Şenel, Soft metric spaces, gaziosmanpas [Ph.D. thesis], University Graduate School of Natural and Applied Sciences Department of Mathematics, 2013.

[22] M. Shabir and M. Naz, "On soft topological spaces," Computers \& Mathematics with Applications, vol. 61, no. 7, pp. 1786-1799, 2011.

[23] I. Zorlutuna, M. Akdağ, W. K. Min, and S. Atmaca, "Remarks on soft topological spaces," Annals of Fuzzy Mathematics and Informatics, vol. 3, no. 2, pp. 171-185, 2012.

[24] J. C. Kelly, "Bitopological spaces," Proceedings of the London Mathematical Society, vol. 13, no. 3, pp. 71-89, 1963.

[25] L. Motchane, "Sur La Notion Diespace Bitopologique et Sur Les Espaces de Baire," Comptes Rendus de l'Académie des Sciences, vol. 224, pp. 3121-3124, 1957.

[26] A. A. Ivanov, "Problems of the theory of bitoplogical spaces," Zap. Nauchn. Sem. Leningrad. Otdel. Mat. Inst. Steklov. (LOMI), vol. 167, no. 6, pp. 5-62, 1988 (Russian), English Translation: Journal of Soviet Mathematics, vol. 52, no. 1, pp. 2759-2790, 1990.

[27] G. C. L. Brümmer, "Two procedures in bitopology," in Categorical Topology: Proceedings of the International Conference, Berlin, August 27th to September 2nd, 1978, vol. 719 of Lecture Notes in Mathematics, pp. 35-43, Springer, Berlin, Germany, 1979.

[28] M. C. Datta, "Projective bitopological spaces," Australian Mathematical Society. Journal. Series A. Pure Mathematics and Statistics, vol. 13, pp. 327-334, 1972.

[29] M. C. Datta, "Projective bitopological spaces, II," Journal of the Australian Mathematical Society, vol. 14, no. 1, pp. 119-128, 1972.

[30] B. P. Dvalishvili, Bitoplogical Spaces; Theory, Relations with Generalized Algebraic Structures, and Applications, vol. 199 of North-Holland Mathematical Studies, Elsevier Science, 2005.

[31] C. W. Patty, "Bitopological spaces," Duke Mathematical Journal, vol. 34, pp. 387-391, 1967.

[32] D. Adnadjević, "Ordered spaces and bitopology," Glasnik Matematički Serija III, vol. 10, no. 30, pp. 337-340, 1975.

[33] B. Banaschewski and G. C. Brummer, "Stably continuous frames," Mathematical Proceedings of the Cambridge Philosophical Society, vol. 104, no. 1, pp. 7-19, 1988.

[34] H. A. Priestley, "Ordered topological spaces and the representation of distributive lattices," Proceedings London Mathematical Society, vol. 24, no. 3, pp. 507-530, 1972.

[35] O. Ravi and M. L. Thivagar, "A bitopological $(1,2)^{*}$ semigeneralised continuous maps," Bulletin of the Malaysian Mathematical Sciences Society, vol. 29, no. 1, pp. 79-88, 2006.

[36] R. E. Smithson, "Multifunctions and bitopological spaces," Journal of Natural Sciences and Mathematics, vol. 11, pp. 191-198, 1971.

[37] S. Hussain and B. Ahmad, "Some properties of soft topological spaces," Computers \& Mathematics with Applications, vol. 62, no. 11, pp. 4058-4067, 2011.

[38] N. Çağman, S. Karatas, and S. Enginoğlu, "Soft topology," Computers \& Mathematics with Applications, vol. 62, no. 1, pp. 351-358, 2011.

[39] G. Şenel and N. Çağman, "Soft topological subspaces," Annals of Fuzzy Mathematics and Informatics, vol. 10, no. 4, pp. 525-535, 2015. 


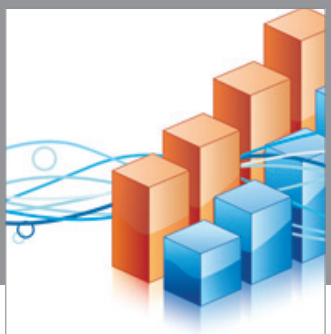

Advances in

Operations Research

vatem alat4

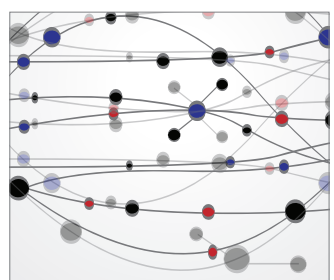

\section{The Scientific} World Journal
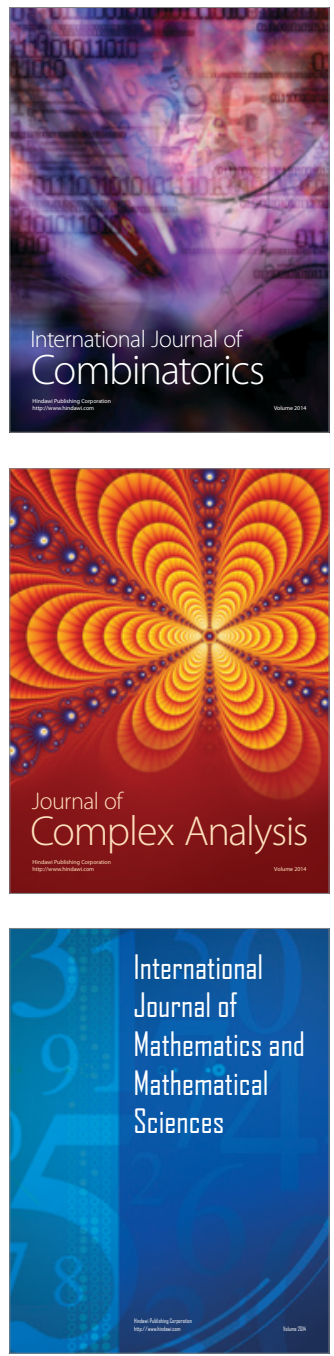
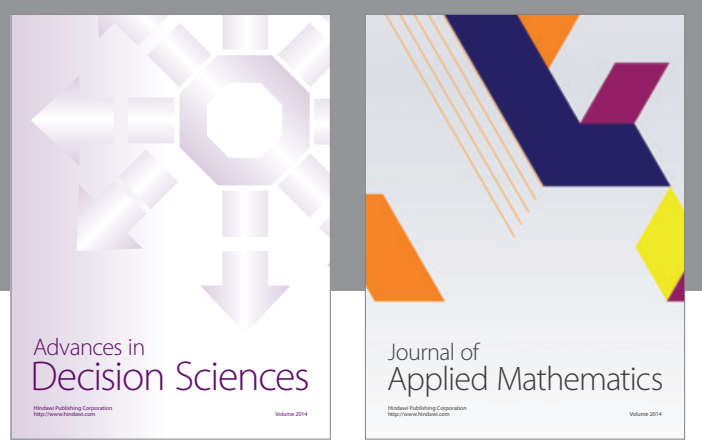

Algebra

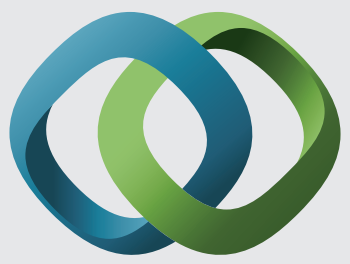

\section{Hindawi}

Submit your manuscripts at

http://www.hindawi.com
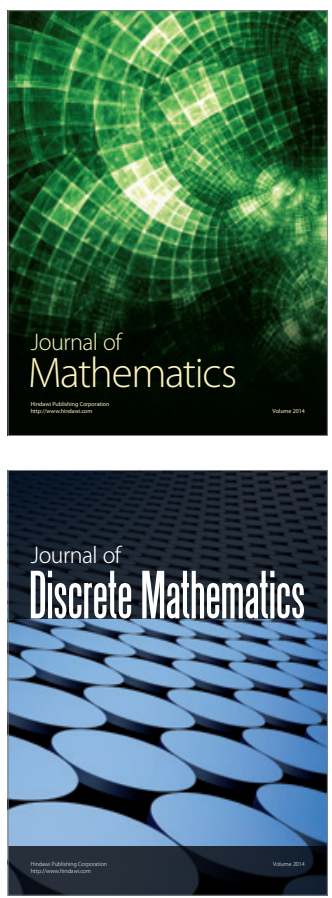

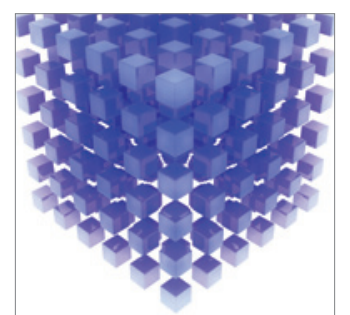

Mathematical Problems in Engineering
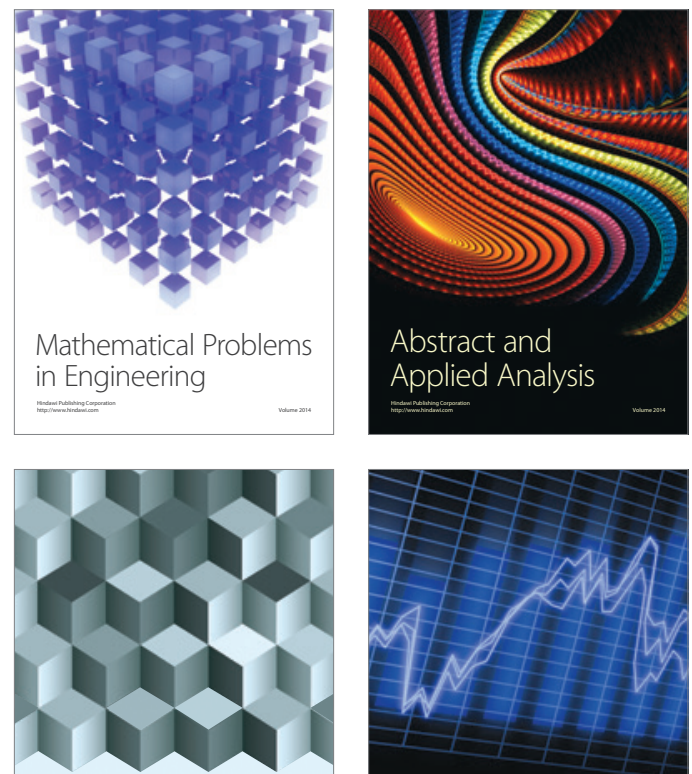

Journal of

Function Spaces

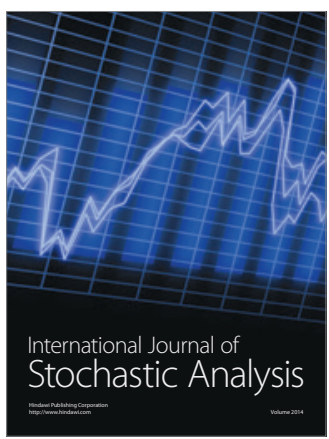

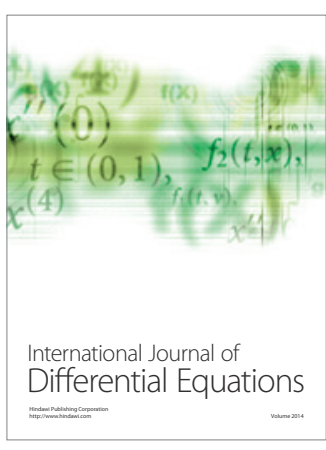
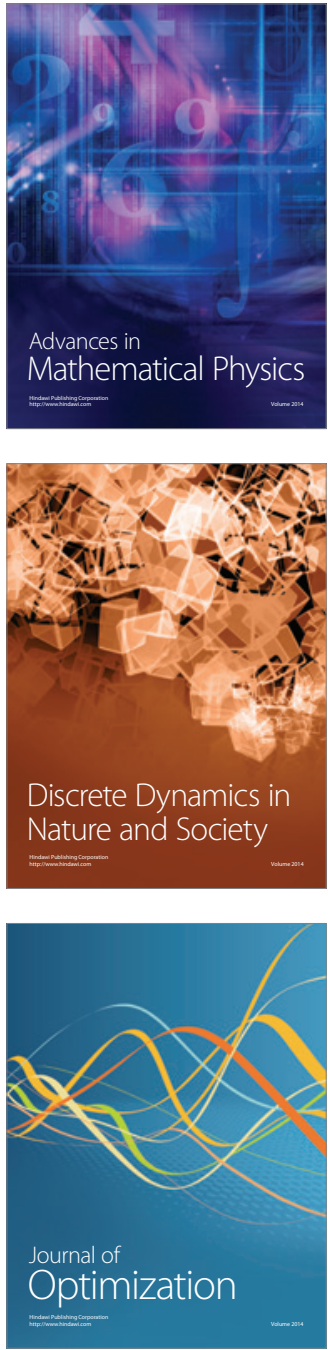